\title{
PNEUMOTHORAX AND PULMONARY INTERSTITIAL EMPHYSEMA IN THE NEWBORN
}

\author{
BY \\ N. E. FRANCE, I. GORDON and F. M. HUMPHRIES \\ From the Mothers' Hospital (Salvation Army) and the Queen Elizabeth Hospital for Children, London
}

(RECEIVED FOR PUBLICATION MAY 28, 1952)

With the greatly increased interest in neonatal morbidity in recent years it is now realized that pneumothorax and pulmonary interstitial emphysema in the newborn period, for many years regarded as medical curiosities, are not uncommon. Indeed, Davis and Stevens (1930) demonstrated the presence of pneumothorax radiologically in $1 \%$ of 702 consecutive babies and Solis-Cohen and Bruck (1934) in approximately $2 \%$ of 500 neonates. While the majority recover spontaneously, in others the condition progresses rapidly and death may ensue.

The following cases illustrate the association between obstructive atelectasis, pulmonary interstitial emphysema and pneumothorax. In addition, two fatal cases of a congenital anomaly of the lungs (pulmonary alveolar dysplasia) are described in both of which pulmonary interstitial emphysema developed. Pulmonary alveolar dysplasia does not appear to have been recorded before as a cause of interstitial emphysema and pneumothorax in the newborn.

\section{Case Reports}

Case 1. This girl was the firstborn of a 36-year-old mother and was delivered by forceps as foetal distress developed at the end of the first stage of labour. The baby weighed 2,965 g. (6 lb. 9 oz.) and was severely asphyxiated at birth. She gasped before mucus catheterization was performed. A good deal of meconiumstained fluid was removed from the pharynx, and intranasal oxygen was given. Regular respirations were established in 10 minutes but the baby was pale and moaning. When 30 minutes old she bled from the umbilical cord and became white and shocked. A transfusion of $60 \mathrm{ml}$. of bank blood (Group $\mathrm{O} \mathrm{Rh}$ negative) was given via the umbilical vein with considerable improvement in her condition although her vigour was slight. She had periods of restlessness and at 63 hours of age she was still pale and slightly cyanosed with a whining cry. There were then signs of left pneumothorax with hyper-resonance and poor air entry, and bronchial breathing and occasional crepitations were heard over the right lung. A radiograph of the chest confirmed the diagnosis of left pneumothorax and showed gross displacement of the heart to the right (Fig. 1).

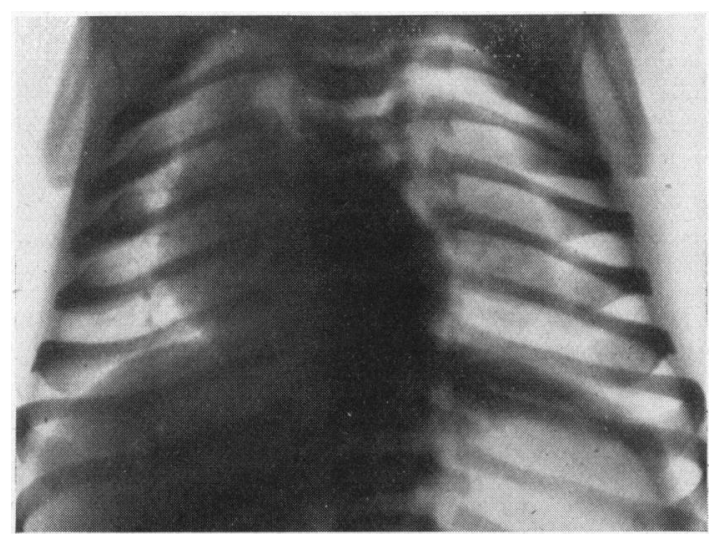

FIG. 1.-Radiograph of Case 1 showing left-sided pneumothorax and displacement of the heart to the right.

Air, $360 \mathrm{ml}$., was withdrawn from the left pleural sac by a needle. It was not under pressure and its removal produced no alteration in the child's condition. Twelve hours later her condition was critical and, in order to exclude the presence of tension pneumothorax, needles were inserted into both pleural cavities but no air was removed. Some hours later the baby was restless and twitching; she produced several blood-stained vomits and a slightly blood-stained vaginal discharge. A further radiograph showed a pneumothorax, now on the right side with a swing of the heart back to the left. The baby was still pale, and slightly cyanosed with gasping breathing. The haemoglobin was $100 \%(14 \cdot 8 \mathrm{~g}$. per $100 \mathrm{ml}$.). She continued to vomit blood and when 4 days old was given a transfusion of fresh blood. In spite of several cyanotic attacks her condition gradually improved, and a radiograph taken at 9 days showed complete absorption of all air from the plearal sacs and, at 17 days, complete pulmonary expansion. Streptomycin, $25 \mathrm{mg}$. intramuscularly four-hourly, was given 
for the first three days of life and penicillin, 50,000 units intramuscularly four-hourly, for 14 days.

SUMmARY. Intra-uterine asphyxia; aspiration of meconium-stained liquor amnii; obstructive atelectasis; probable pulmonary interstitial emphysema; haemorrhagic disease; bilateral pneumothorax; recovery.

Case 2. A boy weighing 3,680 g. (8 lb. 2 oz.) at birth, born at the Mothers' Hospital, was the third child of a 34-year-old mother. Her previous pregnancies were normal but a prolapsed uterus had necessitated an extensive perineal repair. The child was therefore delivered by caesarean section at 38 weeks of gestation. He was limp at birth and responded slowly to mucus catheterization and intranasal oxygen. A considerable amount of mucus drained from the upper respiratory passages for several hours after birth. When 4 hours old he was vigorous and had a good colour although slight rib recession was noted. No adventitious sounds were heard in the chest and there was good air entry over all areas. On the second day of life he was slightly cyanosed and moaning and showed increased rib recession and diminished air entry over both lung fields. Penicillin, 20,000 units, was given intramuscularly every four hours and the baby was placed in an oxygen bell. The following day he was much worse with deep cyanosis, weakness and rapid, grunting respirations. Numerous inspiratory crepitations were heard over the front of the left chest. Nasal and throat swabs produced a profuse growth of Streptococcus haemolyticus. Despite intramuscular injections of streptomycin, $50 \mathrm{mg}$. eight-hourly, death occurred 48 hours after birth.

A post-mortem radiograph showed partial left-sided pneumothorax with gross displacement of the heart to the right. The right lung showed no obvious aeration but the left lung appeared emphysematous (Fig. 2).

NECROPSY FINDINGS. The body was that of a newborn white male weighing $3,630 \mathrm{~g}$. and measuring $53 \mathrm{~cm}$. in length (crown-heel). When the chest was opened

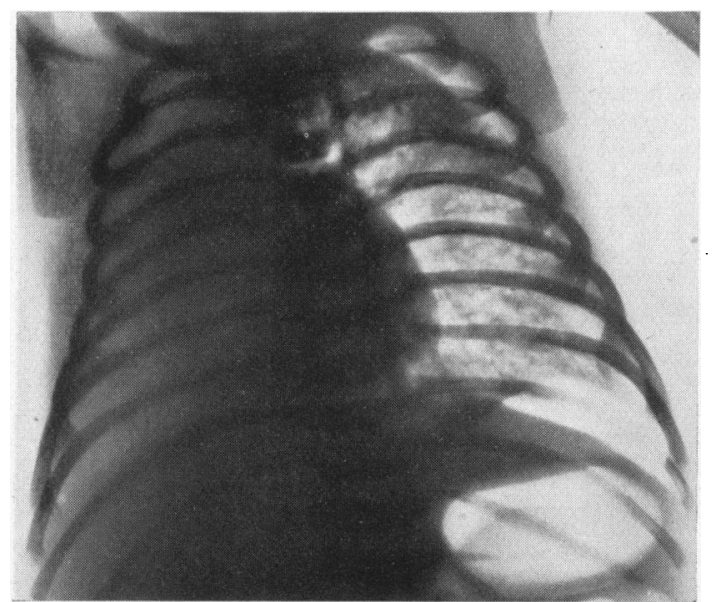

FIG. 2.-Post-mortem radiograph of Case 2 showing left-sided pneumothorax with displacement of the heart to the right, poor aeration of the right lung and emphysema of the left lung. under water a few millilitres of air escaped from the left pleural cavity. The right lung was covered by normal pleura and was generally pink except for the lower lobe posteriorly which was purple and firm. The left lung showed numerous subpleural bullae over both lobes and large numbers of round, air-containing spaces on the cut surface. Most of these bubbles measured about $3 \mathrm{~mm}$. in diameter but others were as large as $6 \times 4 \mathrm{~mm}$. A' haemolytic streptococcus (Group A) was isolated from both the throat and bronchus.

Apart from a few small subarachnoid haemorrhages over the cerebrum, all other organs were normal.

Microscopy. In the right lung most of the alveoli were smaller than normal and contained cornified amniotic cells and

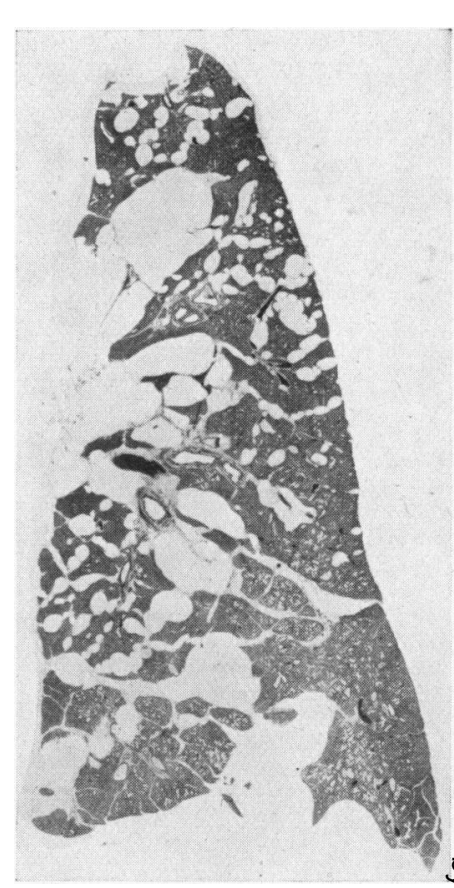

FIG. 3.-Whole lung section (left) of Case 2 showing the distribution of interstitial emphysema in the pulmonary septa.

granular material, frequently associated with a marked inflammatory exudate. Other alveoli were atelectatic.

In addition to the features seen in the right lung there was massive interstitial emphysema in the left lung (Fig. 3). The bubbles of air lay in the connective tissue surrounding the septal blood vessels and extended to the hilum and subpleural tissue. In many places blood vessels were isolated in the centres of air spaces (Fig. 4).

Summary. Aspiration of liquor ammii; obstructive atelectasis; aspiration pneumonia (streptococcal); pulmonary interstitial emphysema; left pneumothorax; death.

Case 3. A boy weighing $2,745 \mathrm{~g}$. (6 lb. $1 \mathrm{oz}$.) at birth, born at the Mothers' Hospital, was the result of the third

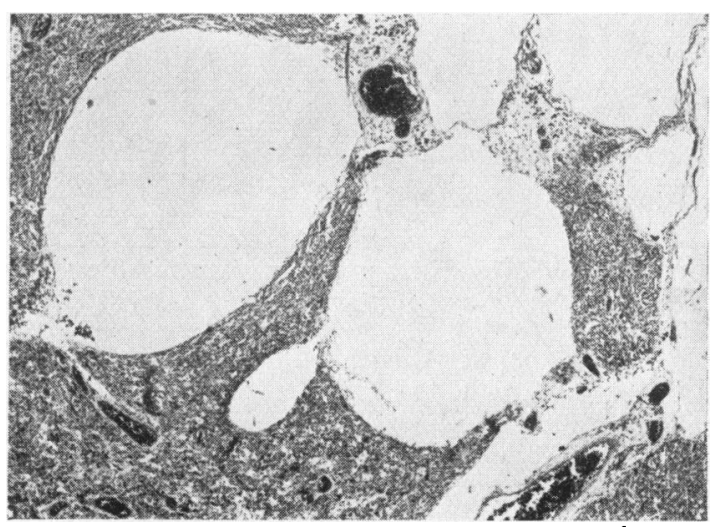

FIG. 4.-Photomicrograph of lung of Case 2 showing large air spaces in the perivascular tissue $(\times 29)$. 
pregnancy of a 31-year-old mother with a generally contracted pelvis and suffering from mitral stenosis. Delivery was by caesarean section at 38 weeks' gestation. The baby's condition was quite satisfactory at birth and remained so until the age of 3 days when he vomited several times and was jaundiced. By the next day he was very ill with cyanosis, rapid breathing, some subcostal recession and a temperature of $104^{\circ} \mathrm{F}$. He was treated with penicillin, 150,000 units daily in divided doses intramuscularly, and with oxygen, and on the following day was much improved. It was noted that the right side of the thorax moved less than the left side and coarse crepitations were heard at the right base. A post-nasal swab produced a pure growth of $B$. friedländer $i$ insensitive to penicillin (20 units per $\mathrm{ml}$.). A radiograph of the thorax showed a right-sided pneumothorax with collapse of all lobes of the lung but no displacement of the heart (Fig. 5). As the child was steadily improving

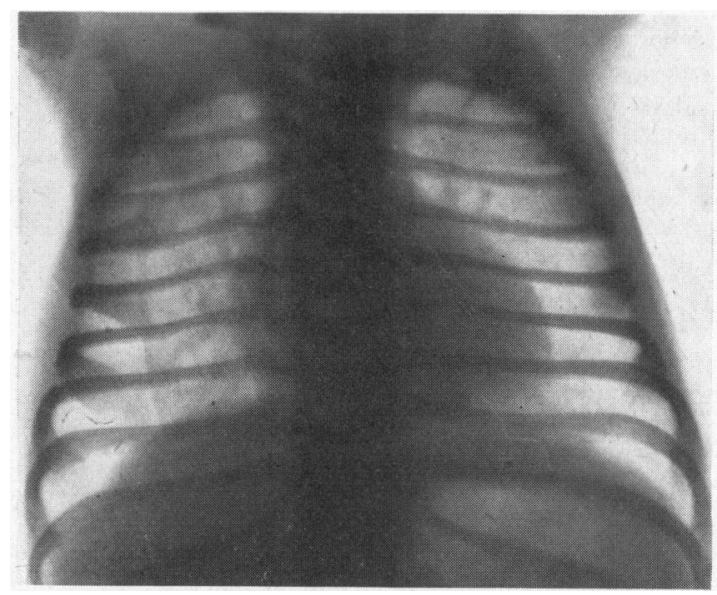

FIG. 5.-Radiograph of Case 3 showing right-sided pneumothorax with no mediastinal displacement.

penicillin therapy was continued for seven days, in spite of the insensitivity of the organism in vitro. The child gained weight and on the seventeenth day after birth a radiograph showed normal lung fields with no evidence of pneumothorax.

Summary. Pneumonia (B. friedländeri); presumably pulmonary interstitial emphysema; right pneumothorax; recovery.

Case 4. This boy, born at home, was the result of the mother's ninth pregnancy and was born eight weeks prematurely following mild ante-partum haemorrhage. At birth he weighed $2,090 \mathrm{~g}$. $(4 \mathrm{lb} .10 \mathrm{oz}$.) and remained well until 16 days old when, half an hour after a feed, he started to scream and to froth at the mouth. He became grey and collapsed but rapidly recovered. He was admitted to the Queen Elizabeth Hospital for Children. No abnormal physical signs were elicited but a radiograph of the thorax 24 hours later showed bilateral partial pneumothoraces without mediastinal displacement (Fig. 6). No specific treatment was given and at 20 days of age the air had all been absorbed. During the next year he

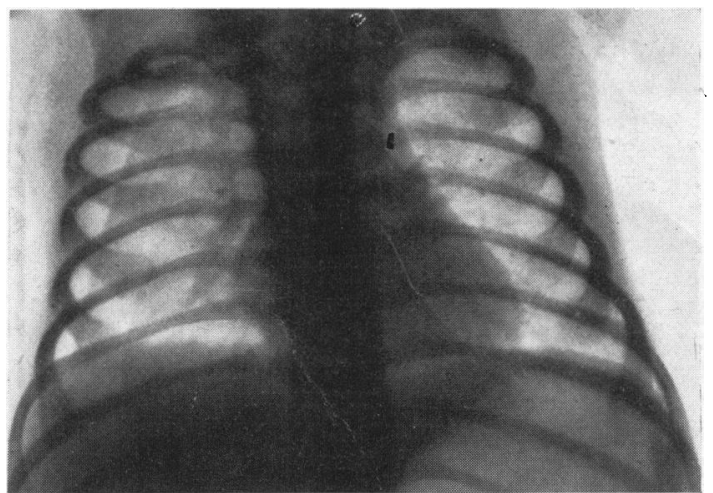

FIG. 6.-Radiograph of Case 4 at 17 days of age showing bilateral pneumothoraces.

suffered from several mild bronchitic attacks. When 16 months old a radiograph of the chest showed left basal pneumonia which, on resolution, revealed a pneumatocoele in the posterior part of the left lower lobe (Fig. 7).

SUMmarY. Antenatal anoxaemia; presumably aspiration of liquor amnii; possibly pulmonary interstitial emphysema; bilateral pneumothorax at 16 days of age; pneumonia and pneumatocoele at 16 months of age; recovery.

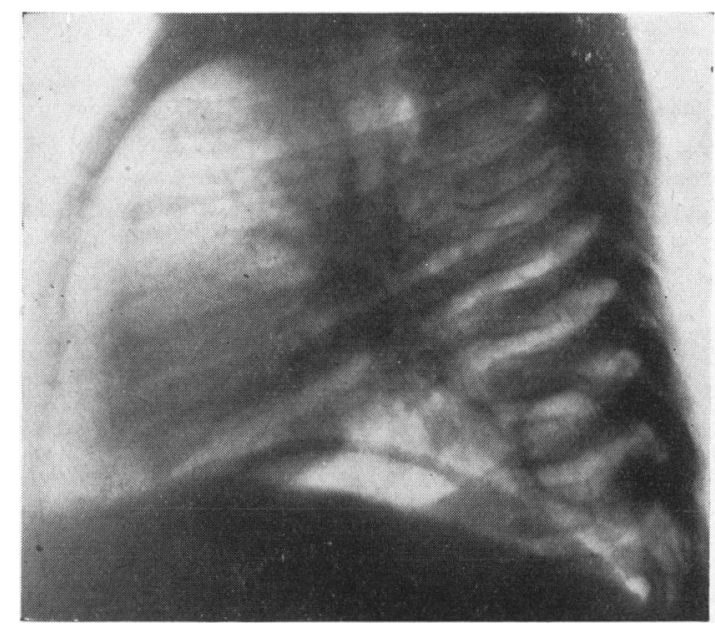

FIG 7.-Radiograph of Case 4 at 16 months of age showing pneumatocoele in the left lower lobe posteriorly.

Case 5. A boy weighing $2,400 \mathrm{~g}$. $(5 \mathrm{lb} .5 \mathrm{oz}$.) at birth was born at the Mothers' Hospital of a primiparous mother aged 22 years. Two attempts at external version were unsuccessful and delivery in the thirtyeighth week of pregnancy was by the breech with extended arms and legs. There was foetal distress and the child was thought to have inspired before the birth of the head. He was limp and cyanosed at birth. When examined he had marked bilateral rib recession 
and coarse crepitations were heard over both lung fields. The abdomen was scaphoid. Blood-stained mucus was aspirated from the pharynx. He was given 20,000 units of penicillin intramuscularly but his condition rapidly deteriorated with very marked cyanosis. He died two and a half hours after birth. A post-mortem radiograph of the thorax showed partial right-sided pneumothorax with displacement of the heart to the left (Fig. 8).

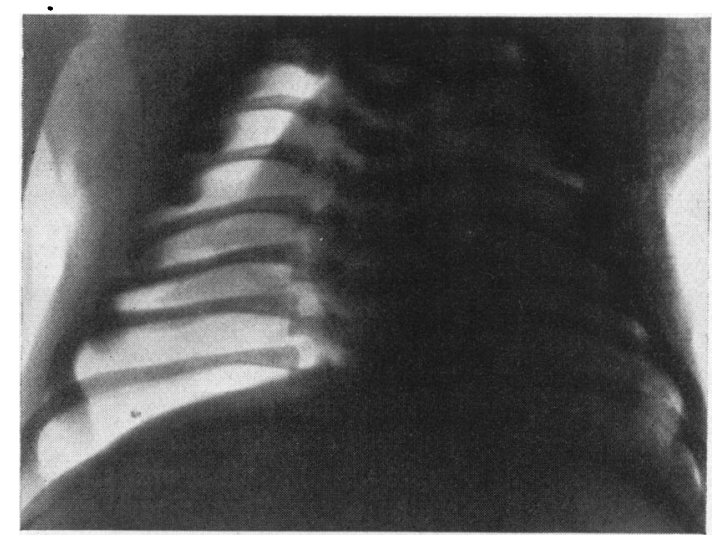

FIG. 8.-Post-mortem radiograph of Case 5 showing right-sided pneumothorax and displacement of the heart to the left.

NeCROPSY Findings. The body was that of a white male weighing $2,400 \mathrm{~g}$. and measuring $50 \mathrm{~cm}$. in length (crown-heel). On opening the thorax under water a number of bubbles issued from the right pleural sac. Both lungs were light purple, had a rubbery consistency and showed numerous subpleural air bubbles up to $5 \mathrm{~mm}$. in diameter over all surfaces. A number of small bubbles were present in the septa on the cut surfaces and these extended into the mediastinum.

Both kidneys were tiny, each measuring $2.4 \times 1.6 \times$ $1.2 \mathrm{~cm}$., and were composed of numerous small cysts

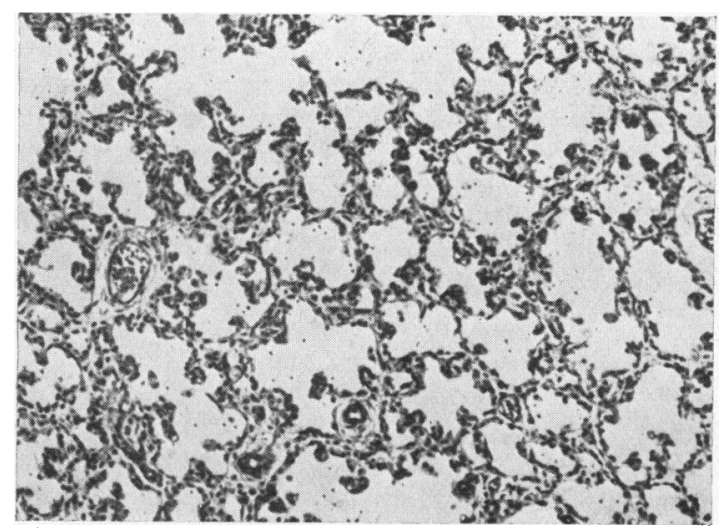

FrG. 9.-Photomicrograph of Case 5 showing the distinctive pattern of the lung in alveolar dysplasia $(\times 90)$. up to $3 \mathrm{~mm}$. in diameter. Both pelves were of normal size but the ureters were grossly dilated. The bladder had an average thickness of $6 \mathrm{~mm}$., the increase being apparently due to thickening of the mucosa which was everywhere firm and rough. There was no evidence of an organic urethral obstruction and the abdominal muscles were normal. The testes lay $4 \mathrm{~cm}$. above the brim of the pelvis.

Microscopy. The lobules of the lung were separated from each other by large spaces in the septa surrounding the blood vessels and representing interstitial emphysema. Although some alveoli were collapsed most were expanded and had a distinctive appearance (Figs. 9 and 10). The alveolar walls were two to three times their normal thickness and were composed largely of loose fibrillary tissue in which cells were few and capillaries almost absent. The alveoli were lined by an incomplete lining of flattened cells with small elongated nuclei. Projecting from the wall into the lumen of each alveolus were three or four small tufts containing one to four capillaries cut in cross section. The capillaries were separated from the alveolar lumen by a thin basement membrane only. The remaining pulmonary elements were not remarkable although bronchioles appeared rather more numerous than usual, suggesting that there was a relative decrease in the amount of parenchymatous tissue.

Both kidneys were composed mainly of fibrous tissue in which were isolated tubules and occasional glomeruli. There were numerous small cysts lined by flattened epithelium and in one area there was a cartilaginous plaque. The ureters were composed of connective and fibrous tissue in which were occasional smooth muscle fibres. The muscle of the bladder was thin and atrophic, the greater part of the wall being composed of dense fibrous tissue in which were small foci of calcification.

Summary. Congenital alveolar dysplasia of lungs; hypoplastic cystic kidneys and mega-ureters; pulmonary interstitial emphysema; right pneumothorax; death.

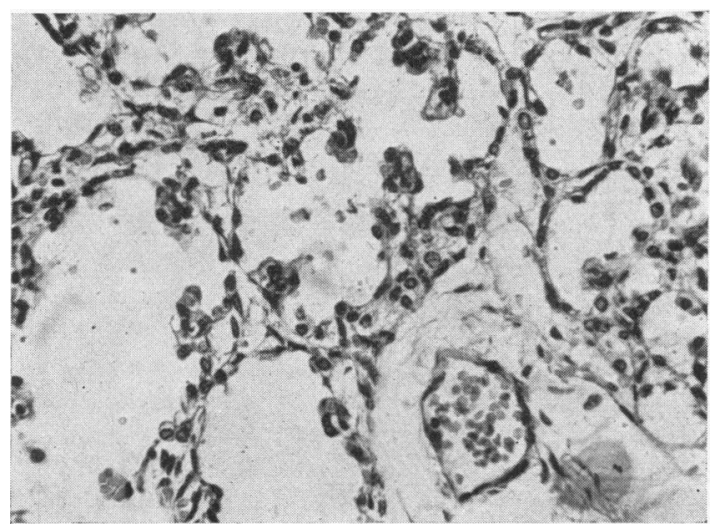

FIG. 10.-Photomicrograph of Case 5 showing the abnormalities of the alveolar walls $(\times 240)$. 
Case 6. A girl, weighing 2,210 g. (4 lb. 14 oz.) at birth, a second child, was born at home in the thirtyseventh week of pregnancy and was delivered by the vertex. There was very little liquor amnii. No evidence of foetal distress was detected but at birth there was white asphyxia. Only a small amount of mucus was obtained on using the mucus catheter. When examined by a doctor 50 minutes after birth she was very limp and pale with feeble, gasping respirations and a slow heart rate. Numerous crepitations were heard anteriorly over both lungs. Her condition rapidly deteriorated and she died in the ambulance on the way to the Mothers' Hospital when one and a half hours old.

On examination after death the abdomen was distended and both kidneys were palpable.

NeCROPSY Findings. The body was that of a premature white female weighing $2,210 \mathrm{~g}$. and measuring $46 \mathrm{~cm}$. in length (crown-heel). There was no evidence of pneumothorax but both lungs showed marked interstitial emphysema both in the subpleural tissue and in the septa and extending to some extent into the mediastinum.

Both kidneys were very large, measuring $8 \times 5 \times 4 \mathrm{~cm}$., and were made up of innumerable tiny cystic spaces containing mucinous fluid. The liver showed patchy fibrosis over the upper surface of the right lobe. Its consistency was firm and the cut surface showed increased fibrous tissue around the larger portal tracts.

Microscopy. The histological picture of the lungs was identical to that of Case 5. The kidneys were composed almost entirely of numerous small cysts lined by a single layer of cuboidal or flattened epithelium. Occasional groups of glomeruli and tubules were present in the interstitial tissue. The liver showed considerable increase in the amount of fibrous tissue around the portal tracts. The bile ducts were numerous, dilated and distorted into bizarre shapes. Many of their lining cells contained granules of haemosiderin.

Summary. Congenital alveolar dysplasia of lungs; polycystic kidneys; cystic fibrosis of liver; pulmonary interstitial emphysema; death.

\section{Discussion}

Pathogenesis of Pneumothorax. The causes of pneumothorax in the newborn infant were summarized by DeCosta (1940) as (1) congenital anomalies, (2) pulmonary infection, (3) trauma, (4) mechanical obstruction and (5) artificial respiration.

The first three of these may be illustrated respectively by broncho-pleural fistula (Wiener, 1930), rupture of a pulmonary abscess or pneumatocoele into a pleural sac (Wilkinson, 1936) and direct trauma to the lung by a fractured bone (Riedweg, 1934, Case 7) or exploring needle, all of which mechanisms are readily understood.

Mechanical obstruction causing pneumothorax in neonates is probably always intrinsic, the bronchi being obstructed by mucus, vernix caseosa, meconium or viscid inflammatory exudate. Extrinsic obstruction such as may be caused by the umbilical cord around the neck (Salmon, Forbes and Davenport, 1947) is probably important only in that it may produce asphyxia with resultant inhalation of amniotic fluid. Obstruction produces patchy over-distension of alveoli by hyperventilation if respiratory efforts are vigorous or as a compensation to areas of collapse. Similarly àlveolar distension may be compensatory to pneumonic consolidation or may be produced by artificial respiration involving the introduction of oxygen under positive pressure into the trachea. Mouth-to-mouth breathing is especially dangerous (Emmert, 1930). It is improbable that over-distension of alveoli can result from normal respiratory movements as was suggested by Strain and Connell (1950) or that intracranial haemorrhage or congenital heart disease (Salmon and Forbes, 1943) are causal factors in alveolar over-distension.

Macklin and Macklin (1944) have demonstrated experimentally that when over-distension of alveoli occurs those alveoli adjacent to blood vessels may rupture and air will then pass into the perivascular connective tissue. Such rupture does not occur when alveoli are completely surrounded by other alveoli or when they lie next to bronchioles, as in such a situation all air-containing passages expand equally and no undue strain is thrown upon the alveolar walls. Once air has escaped into the interstitial tissue it tracks along the vascular sheaths to the hilum and thence into the mediastinum.

Pulmonary interstitial emphysema has important effects on the lung as it tends to splint the lung in a state of inflation and by its perivascular location it interferes with the pulmonary circulation (Macklin and Macklin, 1944). It may cause pulmonary haemorrhage (Salmon, 1947), may assist in the spread of infection (Macklin, 1939) or may rupture into the pleural sac to produce a pneumothorax. Macklin and Macklin (1944) believe that the majority of cases of pneumothorax are produced by the passage of air from the mediastinum into the pleural cavity. Mediastinal emphysema secondary to pulmonary interstitial emphysema produces serious effects by the pressure of air on the heart and great vessels, and they state that when air escapes into the pleural sac, the retroperitoneal tissue or the subcutaneous tissue of the neck it is attended by a relief of symptoms.

Diagnosis of Interstitial Emphysema. The clinical features in cases of established pulmonary interstitial emphysema alone do not appear to have been described although its anatomical features indicate that it may reproduce a similar picture to that of mediastinal emphysema (Salmon, 1947). When the 
latter is present there is dyspnoea and cyanosis with hyperexpansion of the chest, shallow, rapid respirations and inspiratory recession. The physical signs of mediastinal emphysema include reduction of the area of cardiac dullness and weakened heart sounds, the result of displacement of the heart from the anterior chest wall (Biering, 1941). The characteristic crepitations heard during systole, which are a feature of the condition in adults, are not detected in the neonatal period (Hamman, 1939). A postero-anterior radiograph of the chest in mediastinal emphysema may show globules of air just outside the lateral margins of the superior mediastinum, whereas the lateral view shows pools of encapsulated air just beneath the sternum (Gumbiner and Cutler, 1941). Pressure effects may produce radiological evidence of pulmonary congestion. The diagnosis of mediastinal emphysema is assured if subcutaneous emphysema of the neck and upper thorax appears, although this is rare in infancy (McMann and Purcell, 1939).

Clinical Diagnosis of Pneumothorax. Pneumothorax in the newborn presents very different pictures. A small pneumothorax may be found, almost by chance, in a radiograph of a baby who looks quite well, or may occur without much alteration of the picture or physical signs in a baby already acutely ill with respiratory distress. A large pneumothorax is likely to cause severe symptoms and may give striking physical signs although the baby may be so acutely ill that physical examination has to be cut to a minimum. Moreover, even striking physical signs when found may not finally establish a diagnosis without x-ray examination. A bilateral pneumothorax (Case 4) or a pneumothorax associated with pneumonia and causing no displacement of the heart (Case 3) may make the interpretation of signs difficult.

Clinical diagnosis in the neonatal period is notoriously difficult; this is in part due to the fact that essentially similar clinical pictures can be produced by very different pathological conditions as indicated below. In the case of lung conditions there is difficulty in interpreting the significance of the signs elicited in the tiny chest; for example, if there is a difference in resonance to percussion between the two sides, it may not be easy to decide which is abnormal, especially as minor displacements of the heart are difficult to detect with certainty without the help of radiographs. Pneumothorax and diaphragmatic hernia can cause similar signs in the neonatal period. Dyspnoea, cyanosis, perhaps exaggerated respiratory efforts, displacement of the heart, weak or absent breath sounds and diminished movements on the affected side of the chest and even hyper-resonance on this side may be present in both conditions. It is obviously essential to be sure of the correct diagnosis before a needle puncture is made for a supposed pneumothorax. In the differential diagnosis of pneumothorax other conditions to be considered are compensatory over-distension of one lung on account of partial agenesis or failure of expansion of the other lung, and possibly an air-containing pulmonary cyst. Pneumonia, eventration of the diaphragm or a phrenic nerve paralysis can cause respiratory distress, exaggerated respiratory efforts and a difference in percussion note between the two sides of the lower chest, in these cases the less resonant side being the abnormal one.

If some of the pitfalls of diagnosis are borne in mind and one considers the history of the case as well as the physical signs, a clinical diagnosis can be made with some certainty in a proportion of cases of pneumothorax; in others only a radiograph will establish its presence. However, it is the large pneumothorax causing severe symptoms in which the establishment of the diagnosis is urgent and in such cases the history may be of material help.

The Relationship between Obstructive Atelectasis and Pneumothorax. In the history of a pneumothorax the antecedent factors are often intrauterine asphyxia, aspiration of amniotic sac contents and obstructive atelectasis with or without pneumonia.

Five cases of pneumothorax have been described, and of these, three (Cases 1,2 and 4) followed inhalation of fluid before or at birth with consequent obstructive atelectasis, one (Case 3) followed infection with Friedländer's bacillus, possibly causing obstruction of the bronchi with viscid secretion as well as damage to tissues from infection, and one (Case 5) resulted from a congenital anomaly of the alveoli preventing adequate oxygenation of the blood as well as preventing normal expansion of the lungs in response to respiratory efforts. Pulmonary interstitial emphysema is likely to arise when there is patchy obstruction to the entrance of air into the alveoli of an infant who has the vigour to make respiratory efforts of sufficient strength to over-distend and cause the rupture of alveoli; an infant whose respiratory efforts are feeble throughout is not likely to burst alveoli. If we exclude methods of resuscitation probably the commonest cause of pneumothorax in the newborn is inhalation of amniotic sac contents with obstructive atelectasis, interstitial emphysema and the rupture of bullae. Thus, when faced with a possible case of pneumothorax in the newborn, one should inquire into 
factors likely to have produced intra-uterine asphyxia, such as partial separation of the placenta as indicated by ante-partum haemorrhage (Case 4), continuing compression of the placenta by severe and frequent pains or interruption of the foetal circulation by a prolapsed cord; evidence of foetal asphyxia in utero in the shape of change in the foetal pulse rate or the passage of meconium (Case 1); evidence of asphyxia at birth (Cases 1, 2 and 5); signs of obstructive atelectasis with fairly vigorous respiratory efforts and subcostal recession at each inspiration.

White asphyxia, slow establishment of respiration with continuing pallor, cyanosis or cyanotic attacks, shallow, irregular respirations, twitching and restlessness or complete lethargy may betoken intracranial trauma with haemorrhage or may result from asphyxia alone. In either case there will often be obstructive atelectasis, though if breathing is feeble this usually gives no distinctive physical signs and is unlikely to cause interstitial emphysema or pneumothorax. If after birth the baby has sufficient vigour to make good respiratory efforts producing marked subcostal recession, that is the infant who may develop interstitial emphysema and pneumothorax, though with failing vigour the respirations may become shallow again and recessions cease to be present.

The infant (Case 3) who developed a pneumothorax with pneumonia presumably developed interstitial emphysema from similar patchy obstruction of smaller bronchi as that leading to interstitial emphysema in obstructive atelectasis. The relationship between the pneumothorax and the subsequently found pneumatocoele in Case 4 is difficult to assess. Bilateral pneumothoraces, as in this case, are not likely to be produced by rupture of congenital cysts although this has been described by Miller (1926). The process resulting in the rupture of aveoli into the pleural sacs may have produced an encapsulated collection of air in the pulmonary supporting tissue. It is more probable, however, that the pneumatocoele arose independently of the pneumothorax and secondary to an inflammatory process occurring at 16 months of age.

Alveolar Dysplasia and Pneumothorax. Although Case 6 showed no pneumothorax, it is included in the series because the pathology was identical to that of Case 5. Both of these children showed an unusual alveolar pattern which may be described as alveolar dysplasia of a type distinct from that described by MacMahon (1948).

MacMahon's alveolar dysplasia is characterized by the presence of small alveoli separated by thick alveolar walls resembling foetal mesenchyme with an alveolar lining similar to that of mature lung (MacMahon, 1948). His findings have also been described by Kaufman and Spiro (1951) although many pathologists are doubtful of its existence as a pathological entity (Blystad, Landing and Smith, 1951).

The abnormal lungs in the present cases were associated with congenitally cystic kidneys and in one case with cystic changes in the liver. They presented the following pathological features: (1) Although only a few hours old the alveoli were all expanded to about normal size. (2) The total amount of alveolar tissue was small in proportion to the amount of supporting tissue. (3) The alveolar walls were two to three times their normal thickness and were relatively acellular and avascular. (4) The alveoli were incompletely lined by a single layer of flattened endothelial cells. (5) Projecting into the lumen of each alveolus were small tufts of capillaries with no endothelial covering.

It is probable that lungs so affected are incapable of expansion to the size demanded by the bony and muscular development of the thoracic cage with the result that over-distension and rupture of alveoli occur.

The association of hypoplasia of the lungs and bilateral renal agenesis was first described by Potter (1946) who noted also that with renal agenesis deficiency of liquor amnii (compare Case 6) had been recorded. She described the histological picture as showing inadequate parenchymatous development with rudimentary alveoli often lined by cuboidal epithelium and inhibition of capillary ingrowth into the alveolar walls. The lungs thus resembled these of a foetus of a lower gestational age than the one in which they were found. This observation has been confirmed by Leffler (1951) whose photomicrographs show atelectatic, immature lung. However, the possibility remains that if such alveoli were expanded, they would show a similar picture to that seen in the present cases. Potter (1952) states that she observed moderate hypoplasia of the lungs associated with polycystic kidneys. Histologically they resembled the hypoplastic lungs found in cases of renal agenesis. No other reference to pulmonary changes in any way similar to those described in Cases 5 and 6 has been found in the literature.

\section{Summary}

Five cases of pneumothorax and one case of pulmonary interstitial emphysema in the newborn period are described.

The pathogenesis of pulmonary interstitial 
emphysema and its relationship to obstructive atelectasis and pneumothorax are discussed.

The clinical and radiological features of pneumothorax in neonates are discussed.

The pathological features of an unusual type of alveolar dysplasia are described.

We are indebted to Dr. H. M. Mackay for permission to publish Cases $1,2,3,5$ and 6, and for her great help and encouragement. We also wish to thank Dr. $N$. Jacoby for permission to publish Case 4, Dr. C. J. Hodson for his advice on the radiographs, and Dr. B. Levin for his criticism. Cases 2, 5 and 6 form part of an investigation of foetal and neonatal death supported by a British Medical Association Research Scholarship held by one of us (N.E.F.)

Biering, A. (1941). Acta paediat., Uppsala, 28, 367.
Blystad, W., Landing, B. H. and Smith, C. A. (1951). Pediatrics, 8, 5. Davis, C. H. and Stevens, G. W. (1930). Amer. J. Obstet. Gynec., 20, 73.

DeCosta, E. J. (1940). Ibid., 39, 578.

Emmert, F. (1930). Amer. J. Dis. Child., 39, 1268.

Gumbiner, B. and Cutler, M. M. (1941). J. Amer. med. Ass., 117, 2050.

Hamman, L. (1939). Bull. Johns Hopk. Hosp., 64, 1.

Kaufman, N. and Spiro, R. K. (1951). Arch. Path., Chicago, 51, 434. Leffler, R. J. (1951). Amer. J. clin. Path., 21, 752.

Macklin, C. C. (1939). Arch. intern. Med., 64, 913.

Macklin, M. T. and Macklin, C. C. (1944), Medicine, Baltimore, 23, 281 .

MacMahon, H. E. (1948). Amer. J. Path., 24, 919.

McMann, W. and Purcell, C. W. (1939). J. Pediat., 14, 805.

Miller, R. T. (1926). Arch. Surg., Chicago, 12, 392.

Potter, E. L. (1946). J. Pediat., 29, 68.

(1952) Pathology of the Fetus and the Newborn, p. 260. The Year Book Publishers, Chicago.

The Year Book Publishers, Chicago.
Riedweg, J. (1934). Mschr. Kinderheilk., 61, 1.

Salmon, G. W. (1947). New Orleans med. Surg. J., 100, 253.

- and Forbes, G. B. (1943). J. Pediat., 23, 50.

- and Davenport, H. (1947). Ibid., 30, 260.

Solis-Cohen, L. and Bruck, S. (1934). Radiology, 23, 173.

Strain, J. E. and Connell, J. R. (1950). J. Pediat., 36, 495.

Wiener, C. (1930). Arch. Kinderheilk., 91, 14.

Wilkinson, S. J. (1936). Amer. J. Dis. Child., 52, 361. 